USITP-97-03

April, 1997

hep-th/9704051

\title{
A Picture of D-branes at Strong Coupling
}

\author{
Ulf Lindström ${ }^{a 1}$ and Rikard von Unge ${ }^{b / 2}$ \\ a,b) Institute of Theoretical Physics, University of Stockholm \\ Box 6730, S-113 85 Stockholm SWEDEN \\ a) Institute of Physics, University of Oslo \\ Box 1048, N-0316 Blindern, Oslo, NORWAY
}

\begin{abstract}
We use a phase space description to (re)derive a first order form of the Born-Infeld action for $D$-branes. This derivation also makes it possible to consider the limit where the tension of the $D$-brane goes to zero. We find that in this limit, which can be considered to be the strong coupling limit of the fundamental string theory, the world-volume of the $D$-brane generically splits into a collection of tensile strings.
\end{abstract}

\footnotetext{
${ }^{2}$ e-mail address: ul@physto.se

${ }^{2}$ e-mail address: unge@vanosf.physto.se
} 


\section{Introduction}

$D$-branes [1] provide a description of a certain class of string theory solitons. They are BPS states so they are thought to survive in the strong coupling limit. Formally one may investigate what happens with the Born-Infeld description of $D$-branes in this limit. Since the tension of a $D p$-brane is given by the formula

$$
T_{p}=\frac{2 \pi}{g\left(2 \pi \sqrt{\alpha^{\prime}}\right)^{p+1}},
$$

where $\alpha^{\prime}$ is the inverse of the fundamental string tension and $g$ is the dimensionless string coupling $g=e^{\phi}$, the tension of the $D p$-brane goes to zero whenever $g \rightarrow \infty$, i.e. when the fundamental string theory goes to strong coupling']. We thus find that the strong coupling limit is equivalent to the limit where the $D$-brane tension goes to zero.

In this paper we rederive a first order form of the tensile Born-Infeld action, first presented in [6], using a Hamiltonian approach. In the process of this derivation it becomes clear how to take the $T \rightarrow 0$ limit and we thus find a tensionless version of the Born-Infeld action. Somewhat surprisingly, what we find is that when we let the tension of the $D$-brane go to zero the world volume of the brane generically splits into a collection of tensile strings or, in special cases, massless particles, thus leading to a parton picture of $D$ branes in this limit.

The techniques used when taking the $T \rightarrow 0$ limit in this paper are those previously employed in taking this limit in fundamental string theory [2, 8, 9, 11, 12, 14], i.e. in constructing tensionless stringst]

The plan of the paper is as follows: We will first give a summary of our results, then present their derivation and finally discuss the connection to previous work.

\footnotetext{
${ }^{3}$ In principle the tension of the $D$-brane goes to zero also when the fundamental string tension goes to zero. However, the non-trivial dependence of $\alpha^{\prime}$ of the Born-Infeld action makes this limit much more obscure. We will subsequently put $\alpha^{\prime}=\frac{1}{2 \pi}$.

${ }^{4}$ Recently, tensionless strings have also been considered in the context of $D$-branes [3][4]. They arise, e.g., in the compactification of type-IIB to $6 \mathrm{D}$ on a $K_{3}$ when the area of the 2-cycle shrinks to zero size [5]. In this latter context one does not know the dynamics as yet, and the precise relation to the high energy tensionless strings thus remains unclear.
} 


\section{Results}

Some time ago one of the authors showed how to write a first order version of the Born-Infeld action for a bosonic $p$-brane,

$$
S_{B I}^{2}\left(T_{p}\right)=T_{p} \int d^{p+1} \xi \sqrt{-\operatorname{det}\left(\gamma_{i j}+F_{i j}\right)},
$$

where $\gamma_{i j} \equiv \partial_{i} X^{\mu} \partial_{j} X^{\nu} G_{\mu \nu}(X)$ is the induced metric on the world-sheet from a background metric $G_{\mu \nu}$ and $F_{i j}$ is a world-volume field strength, which in this article we will take to be

$$
F_{i j} \equiv \partial_{[i} A_{j]}+B_{i j}
$$

Here $B_{i j} \equiv \partial_{i} X^{\mu} \partial_{j} X^{\nu} B_{\mu \nu}$ is the pull-back of the back ground Kalb-Ramond field. The first order action is [6]:

$$
S_{B I}^{1}(T)=\frac{1}{2} T \int d^{p+1} \xi \sqrt{-s}\left(s^{i j}\left(\gamma_{i j}+F_{i j}\right)-(p-1)\right),
$$

where $s^{i j}$ is a general second rank world-volume tensor, (no symmetry assumed)曰. We take (2.4) to represent the $D$ brane action, thus disregarding the over all dilaton factor $e^{-\phi}$ in the Lagrangian, since it will play no role for our considerations. If one wishes, it is readily reinserted in all our formulae.

In this letter we derive (2.4) from (2.2) via a Hamiltonian formulation. This process also allows us to take the limit $T \rightarrow 0$ and thus obtain a formulation of the tensionless $D$-brane action, which reads

$$
S_{B I}^{1}(0)=\frac{1}{4} \int d^{p+1} \xi\left(E_{1}^{i} E_{1}^{j}-E_{2}^{i} E_{2}^{j}-E_{[1}^{i} E_{2]}^{j}\right)\left(\gamma_{i j}+F_{i j}\right) .
$$

Integrating out the $A_{i}$-field we are left with

$$
\tilde{S}_{B I}^{1}(0)=\frac{1}{4} \int d^{p+1} \xi\left(E_{A} X^{\mu} E_{B} X^{\nu}\left(\eta^{A B} G_{\mu \nu}+\epsilon^{A B} B_{\mu \nu}\right)\right),
$$

where $A, B=0,1, E_{A} \equiv E_{A}^{i} \partial_{i}$ are "degenerate", (for $p>1$ ), zwei-beins corresponding to a $2 D$ Lorentzian "tangent space". In that tangent space the Minkowski metric is $\eta^{A B}$ and $\epsilon^{A B}$ is the epsilon symbol. The form of

\footnotetext{
${ }^{5}$ This form of the action has recently been used by Hull and Abou Zeid to discuss the geometry of $D$-branes [7].
} 
the actions (2.5) and (2.6) clearly show the nature of the strong coupling limit of $D$-branes that we are considering: In this limit the D-brane $d y$ namics is governed by actions that involve a degenerate metric of rank 2 , $\left(\propto E_{A}^{i} E_{B}^{j} \eta^{A B}\right)$. These actions look like tensile string actions. We may thus expect the dynamics of these objects to be given by a "parton" picture with strings as the partons. It is very reminicent of the tensionless string which in a particular gauge is seen to describe a collection of massless particles moving under a certain constraint [8].

The parton picture will be verified in Section 5 , but let us corroborate it a bit here. We want to interpret $E_{A}^{i} E_{B}^{j} \eta^{A B}$ as a metric density $g^{i j}$ for $i, j=0,1$. This is possible since the rank of $E_{A}^{i} E_{B}^{j} \eta^{A B}$ is 2 . Using $M, N=0,1$ to denote the two lowest values of $i, j$ we thus have $\operatorname{det}\left(g^{M N}\right) \equiv g^{-1} \neq 0$. To make contact with the tensile string we want to write $g^{M N} \equiv T \sqrt{-\tilde{g}} \tilde{g}^{M N}$ with the tension $T$ independent of $\xi^{i}, i \neq 0,1$, and $\tilde{g}^{M N}$ an ordinary contravariant metric (from the $2 D$ point of view). Clearly this requires $g^{-1}=-T^{2}$ which means that this is as far as we get without a gauge choice. Also, we must of course make sure that the interpretation is consistent with all the field equations.

As indicated above, the case $p=1$ is a bit special. We will treat that case here while we defer the general case to Section 5 .

Suprisingly, for $p=1$, the action (2.5) gives a unified description of tensile and tensionless ordinary strings.

We rewrite (2.5) as

$$
\left.\tilde{S}_{B I}^{1}(0)=\frac{1}{2} \int d^{2} \xi\left(\mathbf{g}^{i j} \gamma_{i j}+\sqrt{-\mathbf{g}} \epsilon^{i j} F_{i j}\right)\right),
$$

where $\mathbf{g}^{i j}$ is a symmetric second rank tensor density of weight $\frac{1}{2}$ and $\mathbf{g}$ is its determinant. The $A_{i}$ field equations read

$$
\epsilon^{i j} \partial_{j} \mathbf{g}=0
$$

with solution $\mathbf{g}=-\tilde{T}^{2}$, with $\tilde{T}$ a constant of dimension $(\text { length })^{-1}$, thus introducing a new scale in the theory. Using this we define $\tilde{T} \tilde{\mathbf{g}}^{i j} \equiv \mathbf{g}^{i j}$. Since $\operatorname{det} \tilde{\mathbf{g}}^{i j}=-1$, we have $\tilde{\mathbf{g}}^{i j}=\left(\sqrt{-\operatorname{det} g_{k l}}\right) g^{i j}$, with $g^{i j}$ an ordinary metric. Plugging all this back into the action (2.7) yields

$$
S_{F}^{1}(\tilde{T})=-\frac{1}{2} \tilde{T} \int d^{2} \xi\left(\sqrt{-g} g^{i j} \gamma_{i j}+\epsilon^{i j} B_{i j}\right),
$$

\footnotetext{
${ }^{6}$ In conventions where $\sqrt{-g}$ has weight $\frac{1}{2}$.
} 
i.e., the action for a tensile (fundamental) string with string tension $\tilde{T}$ in the $G, B$ background. (Note that the coupling to the $B$-field comes out correct).

For the special case when $\tilde{T}=0$ in the solution to (2.8), the tensor density becomes degenerate and may be written as $\mathbf{g}^{i j}=V^{i} V^{j}$, with $V^{i}$ being contravariant vector desity fields of weight $\frac{1}{4}$. This leads to the action for a tensionless (fundamental) string [9]:

$$
S_{F}^{1}(0)=-\frac{1}{2} \int d^{2} \xi\left(V^{i} V^{j} \gamma_{i j}\right) .
$$

We conclude this section by noting that the unified description of tensile and tensionless strings just described resembles that of [10], although their

starting point is a different formulation of the tensionless string coupled to world-sheet electromagnetism. As will be seen in Section 4, we recover their formulation integrating out some of the Lagrange multipliers.

\section{Derivation}

In this section we present the derivation of (2.4) from (2.2) for arbitrary $p$. We follow the procedure described, e.g., in [9], i.e., derive the momenta, the constraints and then the Hamiltonian. integrating out the momenta from the phase space Lagrangian we then obtain a configuration space action with the lagrange multipliers for the constraints among the variables. We finally identify those multipliers with geometric objects on the world volume.

The generalized momenta that follow from (2.2) are

$$
\begin{aligned}
\Pi_{\mu} & =\frac{T}{2} \sqrt{-\operatorname{det}(\gamma+F)}\left[(\gamma+F)^{-1}\right]^{(0 i)} \partial_{i} X_{\mu}, \\
P^{a} & =\frac{T}{2} \sqrt{-\operatorname{det}(\gamma+F)}\left[(\gamma+F)^{-1}\right]^{[a 0]}, \\
P^{0} & =0 .
\end{aligned}
$$

Using the relations

$$
\begin{aligned}
& {\left[(\gamma+F)^{-1}\right]^{(i k)} F_{k l}+\left[(\gamma+F)^{-1}\right]^{[i k]} \gamma_{k l}=0,} \\
& {\left[(\gamma+F)^{-1}\right]^{(i k)} \gamma_{k l}+\left[(\gamma+F)^{-1}\right]^{[i k]} F_{k l}=2 \delta_{l}^{i},}
\end{aligned}
$$

one finds the primary constraints

$$
\Pi_{\mu} \partial_{a} X^{\mu}+P^{b} F_{a b}=0,
$$




$$
\begin{aligned}
P^{0} & =0, \\
\Pi_{\mu} \Pi^{\mu}+P^{a} \gamma_{a b} P^{b}+T^{2} \operatorname{det}\left((\gamma+F)_{a b}\right) & =0,
\end{aligned}
$$

where $a, b, \ldots=1, \ldots, p$ are transversal indices. The corresponding Hamiltonian is

$$
\begin{aligned}
\mathcal{H}= & P^{i} \partial_{i} A_{0}+\sigma P^{0}+\rho^{a}\left(\Pi_{\mu} \partial_{a} X^{\mu}+P^{b} F_{a b}\right) \\
& +\lambda\left(\Pi_{\mu} \Pi^{\mu}+P^{a} \gamma_{a b} P^{b}+T^{2} \operatorname{det}\left((\gamma+F)_{a b}\right)\right),
\end{aligned}
$$

where $\sigma, \rho^{a}$, and $\lambda$ are Lagrange multiplers for the constraints. Preservation of the constraints (3.13) leads to the secondary "Gauss' law" type constraint

$$
\partial_{i} P^{i}=0
$$

but no tertiary constraints. The final Hamiltonian is just the sum of the constraints, in agreement with the diffeomorphism invariance of the original Lagrangian. The phase space action is

$$
\begin{aligned}
S_{P S}= & \int d^{p+1} \xi\left[\Pi_{\mu} \partial_{0} X^{\mu}+P^{a} F_{0 a}-\sigma P^{0}\right. \\
& -\lambda\left(\Pi_{\mu} \Pi^{\mu}+P^{a} \gamma_{a b} P^{b}+T^{2} \operatorname{det}\left((\gamma+F)_{a b}\right)\right) \\
& \left.-\rho^{a}\left(\Pi_{\mu} \partial_{a} X^{\mu}+P^{b} F_{a b}\right)\right] .
\end{aligned}
$$

Integrating out the momenta yields the configuration space action

$$
\begin{aligned}
S_{C S}= & \int d^{p+1} \xi \frac{1}{4 \lambda}\left[\gamma_{00}-2 \rho^{a} \gamma_{0 a}+\rho^{a} \rho^{b} \gamma_{a b}\right. \\
& +\left(\hat{\gamma}^{-1}\right)^{a b}\left(F_{0 a}-\rho^{c} F_{c a}\right)\left(F_{0 b}-\rho^{d} F_{d b}\right) \\
& \left.-4 \lambda^{2} T^{2} \operatorname{det}\left((\gamma+F)_{a b}\right)\right],
\end{aligned}
$$

where $\hat{\gamma}_{a b}$ is the spacial part of the induced metric $\hat{\gamma}_{a b}=\gamma_{a b}$. To linearize these realtions we introduce additional lagrange multipliers $G^{a}, \theta^{a}, \sigma^{a b}, H_{a b}$ and write the action as

$$
\begin{aligned}
S= & \int d^{p+1} \xi \frac{1}{4 \lambda}\left(\gamma_{00}-2 \rho^{a} \gamma_{0 a}+\rho^{a} \rho^{b} \gamma_{a b}+G^{a} \gamma_{a b} G^{b}\right. \\
& +2 \theta^{a}\left(\left(F_{0 a}-\rho^{b} F_{b a}\right)-G^{b} \gamma_{b a}\right) \\
& \left.-4 \lambda^{2} T^{2}\left(\operatorname{det} H_{a b}+\sigma^{a b}\left(H_{a b}-F_{a b}-\gamma_{a b}\right)\right)\right) .
\end{aligned}
$$


If we integrate out $\theta^{a}, \sigma^{a b}$ we get back to the original model but if we integrate out $G^{a}, H_{a b}$ we get

$$
\begin{aligned}
S= & \int d^{p+1} \xi \frac{1}{4 \lambda}\left(\gamma_{00}-\rho^{a} \gamma_{0 a}+\theta^{a} F_{0 a}-\rho^{a} \gamma_{a 0}-\theta^{a} F_{a 0}\right. \\
& +\left(\rho^{a} \rho^{b}-\theta^{a} \theta^{b}+4 \lambda^{2} T^{2} \sigma^{a b}\right) \gamma_{a b} \\
& \left.+\left(\theta^{a} \rho^{b}-\theta^{b} \rho^{a}+4 \lambda^{2} T^{2} \sigma^{a b}\right) F_{a b}+4 \lambda^{2} T^{2}(p-1) H\right),
\end{aligned}
$$

where $H$ is implicitly defined in terms of $\sigma^{a b}$ through the relation

$$
\sigma^{a b}=-\operatorname{det}(H)\left(H^{-1}\right)^{b a} \text {. }
$$

With the identification

$$
\begin{aligned}
s^{00} & =-\frac{1}{4 \lambda^{2} T^{2} H}, \\
s^{0 a} & =\frac{\rho^{a}-\theta^{a}}{4 \lambda^{2} T^{2} H}, \\
s^{a 0} & =\frac{\rho^{a}+\theta^{a}}{4 \lambda^{2} T^{2} H}, \\
s^{a b} & =-\frac{\left(\rho^{a}+\theta^{a}\right)\left(\rho^{b}-\theta^{b}\right)}{4 \lambda^{2} T^{2} H}-\frac{\sigma^{a b}}{H},
\end{aligned}
$$

we may finally write the action as

$$
S=-\frac{T}{2} \int d^{p+1} \xi \sqrt{-s}\left[s^{i k}\left(\partial_{i} X^{\mu} \partial_{k} X_{\mu}+F_{i k}\right)-(p-1)\right] .
$$

\section{The tensionless theory}

The action in (3.19) is suitable for taking the tension to zero. Doing that we obtain the action

$$
\begin{aligned}
S= & \int d^{p+1} \xi \frac{1}{4 \lambda}\left(\gamma_{00}-2 \rho^{a} \gamma_{0 a}+2 \theta^{a} F_{0 a}\right. \\
& \left.+\left(\rho^{a} \rho^{b}-\theta^{a} \theta^{b}\right) \gamma_{a b}+\left(\theta^{a} \rho^{b}-\theta^{b} \rho^{a}\right) F_{a b}\right) .
\end{aligned}
$$

Just as for the tensionless string [9], there are several formulations that may be derived from (4.23). Some are easy to analyse from a geometrical point of view, and we now turn to these. The action (4.23) may be written

$$
S=\frac{1}{4} \int d^{p+1} \xi V^{i} W^{j}\left(\gamma_{i j}+F_{i j}\right),
$$


where we identify

$$
\begin{aligned}
V^{i} & \equiv \frac{1}{\sqrt{\lambda}}\left(1,-\left(\theta^{a}+\rho^{a}\right)\right), \\
W^{i} & \equiv \frac{1}{\sqrt{\lambda}}\left(1,\left(\theta^{a}-\rho^{a}\right)\right) .
\end{aligned}
$$

The Lagrange multipliers have been interpreted as vector densities (of weight $\frac{1}{4}$ ) to ensure diffeomorphism invariance. Note that the rank of $V^{i} W^{j}$ is 2, which explains why $p=1$ is a special case (treated in Section 2). In what follows, we will be concerned with $p>1$. For the tensionless fundamental string a formulation analogous to (4.24) has been extensively used, e.g., for constructing spinning models both in components, [11], and in a superspace formulation [12]. In the those cases it was useful to interpret the vector densities as a degenerate vielbeins. We will elaborate on this interpretation for the present case below.

The degrees of freedom add up when going from (4.23) to (4.24), due to the invariance of (4.24) under the local scale transformations

$$
V^{i \prime}=\Lambda(\xi) V^{i}, \quad W^{i \prime}=\Lambda^{-1}(\xi) W^{i} .
$$

The equations of motion that follow from variation of (4.24) with respect to $W^{i}, V^{i}, A_{i}$ and $X^{\mu}$ are

$$
\begin{aligned}
V^{i}\left(\gamma_{i k}+F_{i k}\right) & =0, \\
\left(\gamma_{i k}+F_{i k}\right) W^{k} & =0, \\
\partial_{i}\left(V^{[i} W^{k]}\right) & =0, \\
\partial_{i}\left(V^{(i} W^{k)} \partial_{k} X^{\mu}\right) & =0,
\end{aligned}
$$

(For more clarity and without loss of generality we have put $G_{\mu \nu}=\eta_{\mu \nu}$ ). The first (and second) show that $\gamma_{i j}+F_{i j}$ has null-eigenvectors. This means that it is degenerate and has zero determinat. Unlike the usual case [9], this does not mean that the world volume is a null surface, however. It merely implies a relation between the $X^{\mu}$ and the $A_{i}$ fields. We may think of the dynamics of the world-volume vector fields as being determined by the coordinate fields. We will analyse the equations further in the next section.

Before doing this let us note yet another form for the action bringing out the geometrical content even better. Defining

$$
V^{i} \equiv E_{1}^{i}+E_{2}^{i}, \quad W^{i} \equiv E_{1}^{i}-E_{2}^{i},
$$


we rewrite (4.24) as described in Section 2:

$$
S=\frac{1}{4} \int d^{p+1} \xi\left(E_{1}^{i} E_{1}^{j}-E_{2}^{i} E_{2}^{j}-E_{[1}^{i} E_{2]}^{j}\right)\left(\gamma_{i j}+F_{i j}\right) .
$$

Integrating out the $A_{i}$-field we are left with

$$
S=\frac{1}{4} \int d^{p+1} \xi\left(E_{A} X^{\mu} E_{B} X^{\nu}\left(\eta^{A B} G_{\mu \nu}(X)-\epsilon^{A B} B_{\mu \nu}(X)\right),\right.
$$

where $A, B, .$. are $2 D$ Lorentz indices, as described in Section 2 . The symmetry (4.26) gets replaced by $2 D$ local Lorentz symmetry in this formulation. In fact, we may view (4.24) as the action (2.5) expressed in $2 D$ light-cone coordinates, as will be further discussed below.

As a final general comment on the geometrical formulation we note that connections for diffeomorphisms, the scale transformations (4.26) or local Lorentz transformations are in general needed for a covariant description of the models. We will not need them here, but note that the analogous objects were very useful for formulating a degenerate version of the 2D supergravity in 2D superspace to describe spinning version of the tensionless string [12].

As an alternative to the above description, (and to make contact with [10]), we now integrate out various fields in the action (4.23). The equation of motion for $\theta^{a}$, using the spacial part of the metric $\hat{\gamma}_{a b}=\gamma_{a b}$, is

$$
\theta^{a}=\hat{\gamma}^{a b}\left(F_{0 b}+\rho^{c} F_{b c}\right)
$$

which leaves us with

$$
\mathcal{L}=\frac{1}{4 \lambda}\left(\gamma_{00}-2 \rho^{a} \gamma_{0 a}+\rho^{a} \rho^{b} \gamma_{a b}+\left(F_{0 a}+\rho^{c} F_{a c}\right) \hat{\gamma}^{a b}\left(F_{0 b}+\rho^{d} F_{b d}\right)\right) .
$$

Continuing to integrate out $\rho^{a}$ we find an equation of motion

$$
\rho^{a}=\left(\hat{M}^{-1}\right)^{a b} M_{b 0}
$$

where we have introduced the matrix $M$ defined as $M_{i k}=\gamma_{i k}-F_{i a} \hat{\gamma}^{a b} F_{b k}$ and its spacial part $\hat{M}_{a b}=M_{a b}$. Inserting this into the Lagragian we find

$$
\mathcal{L}=\frac{1}{4 \lambda}\left(M_{00}-M_{0 a}\left(\hat{M}^{-1}\right)^{a b} M_{b 0}\right) .
$$


Now, by introducing the matrices $N_{i k}=(\gamma+F)_{i k}$ and $K_{i k}=(\gamma-F)_{i k}$ and their spacial parts $\hat{N}$ and $\hat{K}$ we can rewrite this as

$$
\begin{aligned}
\mathcal{L}= & \frac{1}{4 \lambda}\left(\gamma_{00}-F_{0 a}\left(\hat{\gamma}^{-1}\right)^{a b} F_{b 0}\right. \\
& -\left(N_{0 a}-F_{0 e}\left(\hat{\gamma}^{-1}\right)^{e f} N_{f a}\right)\left(\hat{N}^{-1}\right)^{a b} \gamma_{b c} \\
& \left.\times\left(\hat{K}^{-1}\right)^{c d}\left(K_{d 0}+K_{d g}\left(\hat{\gamma}^{-1}\right)^{g h} F_{h 0}\right)\right)
\end{aligned}
$$

which can be rewritten as

$$
\begin{aligned}
\mathcal{L}= & \frac{1}{4 \lambda}\left(\gamma_{00}-N_{0 a}\left(\hat{N}^{-1}\right)^{a b} F_{b 0}\right. \\
& \left.+F_{0 a}\left(\hat{K}^{-1}\right)^{a b} K_{b 0}-N_{0 a}\left(\hat{N}^{-1}\right)^{a b} \gamma_{b c}\left(\hat{K}^{-1}\right)^{c d} K_{d 0}\right) .
\end{aligned}
$$

Using the properties of these matrices under transposition one can simplify this expression

$$
\mathcal{L}=\frac{1}{4 \lambda}\left(\gamma_{00}-N_{0 a}\left(\hat{N}^{-1}\right)^{a b} N_{b 0}\right)
$$

wich is the same as

$$
\mathcal{L}=\frac{\operatorname{det}(N)}{4 \lambda \operatorname{det}(\hat{N})},
$$

and by a suitable redefinition of the lagrange multiplier we arrive at the Lagrangian

$$
\mathcal{L}=V \operatorname{det}(\gamma+F),
$$

which is the tensionless limit of the Born-Infeld action used in 10, 13.

\section{Solution}

We will now study the solutions to the equations (4.27) and show that these describe a collection of tensile strings. The first two equations of motion (4.27) can be reduced to the equations

$$
\begin{aligned}
V^{i} V^{k} \gamma_{i k} & =0 \\
W^{i} W^{k} \gamma_{i k} & =0
\end{aligned}
$$


which say that $V$ and $W$ are null-like vector fields in the induced metric. The third equation can be written as $[V, W]^{i}=(\partial \cdot W) V^{i}-(\partial \cdot V) W^{i}$, which, after choosing the gauge $\partial \cdot V=\partial \cdot W=0$ says that $V$ and $W$ commute and define good coordinates. The coordinates thus defined coordinatize two dimensional submanifolds of the world volume. They will be the world sheets of the constituent strings. On each world sheet we have the differential operators

$$
\begin{aligned}
& \partial_{+}=V^{i} \partial_{i}, \\
& \partial_{-}=W^{i} \partial_{i} .
\end{aligned}
$$

Using these and the gauge choice we see that the equations of motion reduce to

$$
\begin{aligned}
\gamma_{++} & =0, \\
\gamma_{--} & =0, \\
\partial_{+} \partial_{-} X^{\mu} & =0,
\end{aligned}
$$

which are exactly the equations of motion for a string in light cone gauge. The additional coordinate dependence of $X^{\mu}$ now becomes a label distinguishing between different string world sheets.

For the special case when $V^{i}$ and $W^{i}$ are parallel, an analogous analysis shows that the world volume splits into a collection of massless particles.

\section{Discussion}

Rederiving the first order action for Born-Infeld theories using a Hamiltonian approach, we found a direct way of investigating the strong coupling limit of $D$-branes by formally taking the zero tension limit. For $p=1$ this gave us a unified description of tensile and tensionless strings reminicent of that proposed in [10], but here derived as a limit of an underlying description. For $p>1$ an interesting structure emerged. In this limit the $D$-branes may be described as composed of tensile strings. The various strings are then labeled by the additional world-volume coordinates, and the tension may also depend on those. This limit is described by a novel kind of action involving a degenerate vielbein wich connects the world-volume to a $2 \mathrm{D}$ internal space of Lorentzian signature.

This "parton" picture might be interesting in connection with the Mtheory matrix model [15], where the partons are $D$ 0-branes, corresponding 
to the special case where the vector fields $V^{i}$ and $W^{i}$ are parallel. Perhaps a more interesting candidate is the recently proposed matrix model for type IIB string theory [16]. Since there are no 0-branes in that theory the partons would have to be strings.

The form of the action seems excellently suited for a superspace description, and we plan to return to this question of spinning $D$-branes in the near future.

\section{Acknowledgements}

We are grateful to Eric Bergshoeff, Bo Sundborg and Paul Townsend for discussions. The work of UL was supported in part by NFR grant No. FAA/FU 04038-312 and by NorFA grant No. 96.55.030-O. 


\section{References}

[1] J. Polchinski, Phys. Rev. Lett. 75 (1995) 4724.

[2] J. Isberg, U. Lindström, B. Sundborg and G. Theodoridis Nucl. Phys. B411 (1994) 122;

[3] A. Strominger, Phys. Lett. B383, (1996), 44.

[4] O.J. Ganor and A. Hanany, hep-th/9602120, Nucl. Phys. B474 (1996) 122; A. Hanany and I. R. Klebanov, hep-th/9606136, Nucl. Phys. B482 (1996) 105; O.J. Ganor, hep-th/9605201, Nucl. Phys. B489 (1997) 95, Nucl. Phys. B479 (1996) 197, hep-th/9607092; K. J. Davis, "Mirror symmetry, $\mathrm{N}=1$ superpotentials and tensionless strings on Calabi-Yau four folds" hep-th/9609005; P. Mayr, "A note on tensionless strings in M-theory" hep-th/9610162.

[5] E. Witten, hep-th/9507121; Nucl. Phys. B463, (1996), 383.

[6] U. Lindström, Int.J.of Mod.Phys. A10 (1988) 2401.

[7] M. Abou Zeid and C. M. Hull, "Intrinsic Geometry of D-Branes", hep-th/9704021.

[8] A. Karlhede and U. Lindström, Class. and Quantum Grav. 3 (1986) L73.

[9] U. Lindström, B. Sundborg and G. Theodoridis Phys. Lett. 253B (1991) 319.

[10] E. Bergshoeff, L. A. J. London and P. K. Townsend, Class. Quantum Grav. 9 (1992) 2545.

[11] U. Lindström, B. Sundborg and G. Theodoridis Phys. Lett. 258B (1991) 331.

[12] U. Lindström and M. Roček, Phys. Lett. 271B (1991) 79.

[13] A.A. Tseytlin, Nucl. Phys. B469 (1996) 51.

[14] S. Hassani, U. Lindström and R. von Unge, Class. Quantum Grav. 11 (1994) L79. 
[15] T. Banks, W. Fischler, S.H. Shenker and L. Susskind, "M Theory As A Matrix Model: A Conjecture", hep-th/9610043.

[16] N. Ishibashi, H. Kawai, Y. Kitazawa and A. Tsuchiya, "A Large-N Reduced Model as Superstring", hep-th/9612115. 